\title{
Enfrentamento entre Cuidadores de Pacientes Pediátricos em Tratamento de Leucemia
}

\author{
Marina Kohlsdorf \\ Áderson Luiz da Costa Junior \\ Universidade de Brasília
}

\begin{abstract}
RESUMO
O tratamento do câncer na infância tem acarretado repercussões adversas à família, ao condicionar alterações em práticas parentais e adaptações de rotina. Compreender como os cuidadores pediátricos enfrentam tais desafios constitui um requisito à proposição de intervenções psicossociais mais eficazes, reduzindo os custos comportamentais do tratamento. O objetivo do presente estudo foi investigar as estratégias de enfrentamento, adotadas por 30 cuidadores (média de idade 33,1 anos; DP $=9,4$ ) de crianças e adolescentes, ao longo do semestre inicial de tratamento para leucemias, a partir do uso da Escala Modos de Enfrentamento de Problemas (EMEP) e de entrevista semi-estruturada. A partir do uso de testes estatísticos para associações entre dados demográficos e tipos de estratégias, bem como comparações entre as médias obtidas, os resultados indicam alterações importantes nas estratégias adotadas, tais como o aumento, ao longo do tempo, de estratégias baseadas no problema e diminuição do uso de estratégias focalizadas na regulação emocional. Além disso, foram encontradas associações entre dados sociodemográficos e estratégias de enfrentamento focalizadas na emoção e na busca por suporte social. Os relatos obtidos nas entrevistas mostram outros exemplos de estratégias de enfrentamento além dos itens abordados na EMEP e também as principais demandas e dificuldades associadas ao tratamento. A partir dos resultados, destaca-se que o conhecimento da vivência do cuidador constitui um indicador relevante dos cuidados a serem disponibilizados pelos profissionais de saúde.
\end{abstract}

Palavras-chave: enfrentamento entre cuidadores; câncer; psicologia pediátrica.

\begin{abstract}
Coping Among Pediatric Caregivers During Leukemia Treatment

Pediatric cancer treatment causes adverse repercussions to the family, since it establishes changes in caregiving practices and routine adaptations. Understanding how pediatric caregivers cope with such challenges is a requirement to promote more efficient psychosocial interventions and reducing behavioral costs related to the treatment. The goal of this study was to investigate coping strategies adopted by 30 caregivers (mean age 33,1 years; $\mathrm{SD}=9,4$ ) of children and adolescents, during the first round of leukemia treatment, using an adapted version of a Ways of Coping Questionnaire (Escala Modos de Enfrentamento de Problemas - EMEP) and a semi-structured interview. Using statistical tests for associations between demographic data and types of strategies, as well as comparing the means obtained, the results show important changes in the adopted strategies, such as an increase over time in problem-focused strategies, and a decrease in emotion-focused strategies. Besides that, there were associations between socio-demographic data, emotion-focused coping strategies and the search for social support. The reports from interviews show other examples of coping strategies, besides items in the EMEP, and also the main demands and difficulties associated to this form of treatment. According to the results, it is highlighted that understanding the caregivers' experience constitutes a relevant indicator regarding the care available by health professionals.
\end{abstract}

Keywords: coping among caregivers; cancer; pediatric psychology.

O diagnóstico e tratamento de uma neoplasia na infância podem representar uma série de desafios para o paciente e seus familiares, destacando-se os episódios repetidos de internação, a terapêutica antineoplásica agressiva e acompanhada por efeitos colaterais desagradáveis, a exposição a procedimentos médicos invasivos, o afastamento de atividades sociais e escolares, a incontrolabilidade da resposta clínica, as alterações 
nos cuidados parentais e mudanças na dinâmica familiar, entre outras demandas administradas pelos cuidadores de pacientes pediátricos (Castro \& Piccinini, 2002; Costa Junior, 2005; Hoekstra-Weebers, Jaspers, Kamps \& Klip, 2001; Kohlsdorf, 2008; McGrath, 2002; McGrath, Paton \& Huff, 2005; Motta \& Enumo, 2002; Nascimento, Rocha, Hayes \& Lima, 2005; Young, Dixon-Woods, Findlay \& Heney, 2002).

Geralmente, o manejo de tais desafios é designado aos pais, que, além de acompanhar o processo de adoecimento do filho, têm seu papel estendido, na medida em que precisam administrar as respostas emocionais da criança ou adolescente e de seus irmãos, moderar suas próprias emoções, estabelecer uma relação e comunicação satisfatórias com o contexto médico-hospitalar, adaptar rotinas sociais, ocupacionais e familiares, lidar com ameaças de recaídas, medos e expectativas e ainda zelar pelo bem-estar da criança, com atenção à medicação, intercorrências, efeitos colaterais, estabelecimento de cuidados protetores, acompanhamento em consultas e exames (Clarke, 2004; Clarke \& Fletcher, 2003; Kars, Duijnstee, Pool, Delden \& Grypdonck, 2008; Keegan-Wells \& cols., 2002; McGrath \& cols., 2005; Shiota, Santos \& Miyazaki, 2004; Silva, Pires, Gonçalves \& Moura, 2002).

$\mathrm{Na}$ literatura recente, o estudo do impacto psicossocial do tratamento de câncer pediátrico para os cuidadores, prioritariamente para os pais, inclui a referência a diversos transtornos de comportamento (depressão, ansiedade, sintomas de estresse pós-traumático, vivências de distress) e mudanças funcionais (em práticas educativas, na dinâmica conjugal, na satisfação com suporte social e nos fatores protetores de riscos ao bem-estar); todavia, pouco ainda é conhecido, de forma sistemática e processual, sobre como os pais lidam com o evento do câncer na infância, indicando a necessidade de estudos de acompanhamento que investiguem as mudanças nas estratégias de enfrentamento ao longo da exposição ao tratamento onco-hematológico (Beltrão, Vasconcelos, Pontes \& Albuquerque, 2007; Castro \& Piccinini, 2002; Herman, 2007; Kohlsdorf, 2008; Norberg, Lindblad \& Boman, 2005).

\section{Enfrentamento: caracterização e mensuração}

Tradução para a língua portuguesa do termo em inglês coping, o enfrentamento é definido por Lazarus e Folkman (1984, p. 141) como "esforços cognitivos e comportamentais em constante mudança, com objetivo de manejar (minimizar, evitar ou tolerar) demandas específicas internas e/ou externas que são avaliadas como sobrecarga ou excedendo os recursos pessoais". Neste modelo, Folkman e Lazarus (1985) apontam dois elementos fundamentais: (a) o encontro com o(s) evento(s) estressor(es) não é imediato e estático, mas um processo de interação, relacional, multifacetado, específico às demandas e que ocorre ao longo do tempo; e (b) as estratégias de enfrentamento podem ser aprendidas, modeladas e descartadas.

$\mathrm{Na}$ literatura internacional sobre estratégias de enfrentamento adotadas por cuidadores em contexto onco-hematológico pediátrico, uma parte dos estudos focaliza a investigação de estratégias por meio do relato dos pais (Beltrão \& cols., 2007; Goldbeck, 1998; Greening \& Stoppelbein, 2007; Herman, 2007; Holm, Patterson \& Gurney, 2003; James \& cols., 2002; Munet-Vilaró, 1984; Norberg \& cols., 2005; Patistea, 2005; Patistea, Makrodimitri \& Pantelli, 2000; Rodrigues, Rosa, Moura \& Baptista, 2000), enquanto outros trabalhos focalizam os processos psicológicos indicativos das estratégias de enfrentamento (Clarke-Steffen, 1997; Eiser, Havertnans \& Elser, 1995; Elkin \& cols., 2007; Enskär, Carlsson, Golsäter, Hamrin \& Kreuger, 1997; Felder-Puig, Gallo, Waldenmair, Gadner \& Topf, 2004; Hoekstra-Weebers, Jaspers, Kamps \& Klip, 1998; Klassen \& cols., 2007; Sloper, 2000; Steele, Long, Reddy, Luhr \& Phipps, 2003; Tarr \& Pickler, 1999; Trask \& cols., 2003). Os principais resultados de ambos os tipos de estudo serão descritos a seguir. Cabe ressaltar que, em virtude de diferentes fundamentações teórico-metodológicas, diversas categorizações de estratégias de enfrentamento têm sido referidas na literatura.

Algumas pesquisas indicam o uso intenso de estratégias de enfrentamento focalizadas no problema ou manejo do estressor, que envolvem busca por informações sobre doença e tratamento, controle da medicação e procedimentos médicos, vigilância sobre o estado de saúde da criança, planejamento de metas, cumprimento de recomendações médicas, redefinição cognitiva do problema (por exemplo, comparar a situações piores), organização da rotina, comunicação com a equipe, reorganização de papéis familiares, explicações à criança sobre o tratamento, conformação com a doença e negociações com a equipe médica (Clarke-Steffen, 1997; Eiser, Havertnans \& Elser, 1995; Enskär, Carlsson, Golsäter, Hamrin \& Kreuger, 1997; Goldbeck, 1998; Greening \& Stoppelbein, 2007; Holm, Patterson \& Gurney, 2003; James \& cols., 2002; Munet-Vilaró, 1984; Norberg \& cols., 2005; Patistea, 2005; Rodrigues, Rosa, Moura \& Baptista, 
2000; Steele, Long, Reddy, Luhr \& Phipps, 2003; Tarr \& Pickler, 1999; Trask \& cols., 2003).

Além disso, estratégias de enfrentamento focalizadas no manejo de reações emocionais ao estressor também são referidas pela literatura internacional, incluindo uso de distração, resignação, isolamento social, autocontrole, assistir a filmes, praticar atividades físicas, utilizar medicação antidepressiva, adotar comportamentos de esquiva e fuga, uso de relaxamento, expressão de emoções e protesto, investimento em auto-estima, culpabilização de si e/ou de outros e uso de substâncias psicotrópicas (Clarke-Steffen, 1997; Felder-Puig, Gallo, Waldenmair, Gadner \& Topf, 2004; Greening \& Stoppelbein, 2007; Klassen \& cols., 2007; Munet-Vilaró, 1984; Norberg \& cols., 2005; Patistea, 2005; Rodrigues \& cols., 2000; Tarr \& Pickler, 1999).

Outra categoria de estratégia destacada pela literatura envolve o uso de práticas religiosas e crenças, tais como uso de pensamento positivo, referências à esperança, práticas religiosas de apego à fé e pensamentos fantasiosos (Elkin \& cols., 2007; Enskär \& cols., 1997; Goldbeck, 1998; Greening \& Stoppelbein, 2007; Munet-Vilaró, 1984; Norberg \& cols., 2005; Patistea, 2005; Patistea, Makrodimitri \& Pantelli, 2000).

Além das classificações descritas, a busca por suporte social (conversar, buscar informações com outros cuidadores e equipe médica, coesão e cooperação familiar) tem sido apontada pela literatura como outra estratégia de enfrentamento relevante. As principais fontes de suporte incluem familiares, amigos, cônjuge, equipe de saúde e outros pais em situação similar (Eiser \& cols., 1995; Enskär \& cols., 1997; Felder-Puig \& cols., 2004; Greening \& Stoppelbein, 2007; James \& cols., 2002; Klassen \& cols., 2007; Munet-Vilaró, 1984; Norberg \& cols., 2005; Patistea, 2005; Patistea \& cols., 2000; Tarr \& Pickler, 1999).

De acordo com a literatura internacional, perturbações psicológicas, tais como ansiedade, depressão, estresse pós-traumático e vivências de medo, têm sido associadas ao maior uso de estratégias focalizadas na emoção, uso menos frequente de estratégias focalizadas no problema e percepção de baixo suporte social (Greening \& Stoppelbein, 2007; Hoekstra-Weebers, Jaspers, Kamps \& Klip, 1998; Klassen \& cols., 2007; Norberg \& cols., 2005; Sloper, 2000). Por outro lado, o uso de práticas religiosas e pensamentos positivos, além da satisfação com o suporte social, têm sido associados de forma inversa a manifestações de ansie- dade e depressão (Elkin \& cols., 2007; Greening \& Stoppelbein, 2007).

Recentemente, alguns estudos nacionais, descritos a seguir, têm investigado as estratégias de enfrentamento adotadas por pais de crianças em tratamento de patologias onco-hematológicas, embora pouco tenha sido sistematizado sobre este tema entre os pesquisadores brasileiros. O estudo de Herman (2007), por exemplo, teve como objetivo descrever as práticas parentais e estratégias de enfrentamento adotadas por oito cuidadores de crianças com câncer que participaram de um programa educativo cognitivo-comportamental. Para tanto, foi utilizada a Escala Modos de Enfrentamento de Problemas - EMEP (Seidl, Tróccoli $\&$ Zannon, 2001). A autora descreve que as estratégias de enfrentamento mais referidas na fase de pré-intervenção foram práticas religiosas e, na fase de pósintervenção, destacaram-se as estratégias focalizadas no problema. $\mathrm{Na}$ fase de pós-intervenção, também houve aumento pela busca de suporte social e redução de estratégias focalizadas em emoção e religiosidade.

O estudo de Beltrão e cols. (2007) investigou as estratégias de enfrentamento de mães, em seguida à comunicação do diagnóstico. As estratégias relatadas incluíram a busca de informações, formação de rede de apoio social (família, equipe de saúde e outras mães acompanhantes e amigos) e apego a crenças religiosas. Um recurso social amplamente relatado foi a obtenção de apoio de outras mães, proporcionando maior convivência e compartilhamento de experiências entre pessoas expostas a contingências semelhantes.

R. A. Fernandes, A. R. R. Santos e M. C. O. S. Miyazaki (comunicação pessoal, 13 de fevereiro de 2008) investigaram o uso de estratégias de enfrentamento adotadas por 22 pais de crianças até 12 anos, com diversos tipos de diagnóstico onco-hematológico, também utilizando a Escala Modos de Enfrentamento de Problemas (EMEP), descrita anteriormente. Os resultados mostraram que as estratégias mais adotadas concentraram-se em práticas religiosas, estratégias focalizadas na resolução de problemas e busca por suporte social, seguidas por estratégias focalizadas na emoção.

Os estudos descritos indicam a necessidade de compreender como os cuidadores de pacientes pediátricos em tratamento onco-hematológico lidam com as dificuldades associadas ao tratamento, em especial que tipos de estratégias de enfrentamento são adotadas pelos pais, a fim de possibilitar intervenções mais eficientes neste contexto de tratamento. 


\section{Objetivo}

Considerando que os primeiros seis meses de tratamento para leucemias correspondem à etapa mais crítica do tratamento (em que a terapêutica antineoplásica é mais agressiva, as internações são mais frequentes e prolongadas e a rotina e dinâmica familiares precisam ser imediatamente ajustadas), o objetivo do presente estudo foi investigar as estratégias de enfrentamento adotadas por cuidadores de crianças e adolescentes, bem como mudanças no uso de tais estratégias, ao longo do semestre inicial de tratamento, a partir de uma pesquisa de acompanhamento. Como objetivo secundário, foram investigadas possíveis associações entre dados sociodemográficos dos participantes e a preferência por categorias de enfrentamento.

\section{MÉTODO}

\section{Participantes}

Participaram do estudo 30 cuidadores (cinco pais, 23 mães, uma tia e uma avó) de crianças e adolescentes diagnosticados com leucemia e atendidos no Núcleo de Onco-hematologia da Secretaria de Estado da saúde do Distrito Federal. A média de idade foi 33,1 anos $(D P=9,4)$ e a escolaridade variou entre o segundo ano do ensino fundamental e o ensino superior completo. Em relação aos pacientes acompanhados, a média de idade foi 7,05 anos $(D P=5,17)$. A Tabela 1 apresenta características sociodemográficas dos participantes.

\section{TABELA 1}

Características Sociodemográficas dos Participantes

\begin{tabular}{ll}
\hline Características dos cuidadores ao início do tratamento & Frequência \\
\hline Idade do cuidador & 12 \\
20 a 30 anos & 13 \\
31 a 40 anos & 2 \\
41 a 50 anos & 3 \\
51 a 60 anos & $33,1(9,4)$ \\
MÉDIA (DP) & \\
Escolaridade do cuidador & 8 \\
Ensino fundamental até $4^{a}$. Série completa & 8 \\
Ensino fundamental entre 4a. Série e 8a. Série & 5 \\
Ensino Médio incompleto & 6 \\
Ensino Médio completo & 1 \\
Superior incompleto & 2 \\
Superior completo & \\
Estado civil do cuidador & \\
Solteiro, divorciado ou viúvo & 9 \\
Casado ou união estável & 21 \\
& \\
Renda mensal familiar* & \\
A1: até $R \$ 17.403,00$ & 0 \\
A2: até $R \$ 7.846,00$ & 0 \\
B1: até R\$ $4.461,00$ & 1 \\
B2: até $R \$ 2.397,00$ & 6 \\
C: até $R \$ 1.370,00$ & 9 \\
D: até $R \$ 776,00$ & 8 \\
E: até $R \$ 401,00$ & 6 \\
Dias decorridos entre a internação e a comunicação do diagnóstico** & \\
Até uma semana & \\
Entre 8 e 15 dias & 18 \\
Mais de 16 dias & 4 \\
\hline
\end{tabular}

Nota:

* Conforme critérios adotados pelo Instituto Brasileiro de Geografia e Estatística (IBGE).

** Consequente a dificuldades na realização dos exames diagnósticos e atrasos na entrega de material coletado (em decorrência de greves em órgãos estatais), houve diferenças entre dias decorridos entre internação e comunicação do diagnóstico. 


\section{Instrumentos}

Para o presente estudo, foram utilizados os seguintes instrumentos:

(1) Questionário sociodemográfico: elaborado especialmente para o estudo e utilizado para caracterização dos cuidadores e pacientes em termos de perfil sociodemográfico.

(2) Escala Modos de Enfrentamento de Problemas - EMEP (Seidl \& cols., 2001): avalia estratégias de enfrentamento cognitivas e/ou comportamentais adotadas frente a um estressor, a partir de respostas em escala de cinco pontos $(1=$ eu nunca faço isso; $2=\mathrm{eu}$ faço isso um pouco; $3=\mathrm{eu}$ faço isso às vezes; $4=\mathrm{eu}$ faço isso muito; $5=$ eu faço isso sempre). A Escala compõe-se por 45 itens, distribuídos em quatro fatores: (a) enfrentamento focalizado no problema (alpha de Cronbach $=0,84$ : estratégias voltadas para o manejo e reavaliação do problema); (b) enfrentamento focalizado na emoção (alpha de Cronbach $=0,81$ : estratégias que envolvem esquiva, negação, pensamento irrealista, atribuição de culpa, afastamento do problema e funções paliativas frente ao estressor); (c) enfrentamento baseado na busca de práticas religiosas e pensamento fantasioso (alpha de Cronbach $=0,74$ : estratégias caracterizadas como comportamentos religiosos, bem como pensamentos fantasiosos); (d) enfrentamento baseado na busca por suporte social (alpha de Cronbach = 0,70: procura por apoio social e busca por informação).

(3) Roteiro para entrevista semi-estruturada: elaborado para este estudo, a fim de obter retrospectivamente informações sobre demandas e dificuldades específicas do tratamento e investigar estratégias de enfrentamento não referidas na EMEP.

\section{Procedimento}

O estudo foi aprovado pelo Comitê de Ética em Pesquisa (CEP) da Faculdade de Ciências da Saúde da Universidade de Brasília, sob o Protocolo 142/2006, e a pesquisa desenvolveu-se em três fases. A primeira etapa foi realizada na semana subsequente à comunicação do diagnóstico de leucemia e consequente início do tratamento $(M=3$ dias; $D P=2,42)$. Após convite para participar do estudo, assinatura do Termo de Consentimento Livre e Esclarecido e preenchimento do questionário sociodemográfico, o participante foi solicitado a responder à EMEP.

A fase seguinte foi realizada no próprio Núcleo de Onco-hematologia da Secretaria de Estado da saúde do Distrito Federal, entre dois e três meses desde o início do tratamento $(M=68$ dias; $D P=15,15)$. Após consultas pediátricas de rotina ou durante período de internação, os participantes foram solicitados a responder a EMEP e, a seguir, a participar da primeira entrevista. Na terceira fase, implementada entre cinco e seis meses após o início do tratamento $(M=163$ dias; $D P=16,20)$, os participantes foram, novamente, solicitados a responder à EMEP e a participar da segunda entrevista, etapa também realizada no próprio hospital, após consultas pediátricas de rotina ou durante período de internação.

A análise de dados foi realizada a partir de testes de correlação bivariada de Pearson, para verificar associações entre dados sociodemográficos e estratégias de enfrentamento, além de testes estatísticos de comparações entre médias (como teste $t$ de Student e teste L de tendência central de Page), para verificar a significância estatística dos resultados.

\section{RESULTADOS}

\section{Associações relevantes entre estratégias de enfrentamento, dados sociodemográficos e condição clínica}

Médias relativas a estratégias de enfrentamento, em grupos separados por estado civil (solteiros ou divorciados em comparação a participantes casados ou em relacionamento estável), estiveram bastante semelhantes nos fatores 1, 3 e 4 da EMEP e não houve diferença estatisticamente significativa quanto ao uso de estratégias focalizadas no problema $(t[28]=0,377$; $p=0,712)$, práticas religiosas e pensamentos fantasio$\operatorname{sos}(t[28]=0,367 ; p=0,716)$ ou busca por suporte social $(t[28]=0,285 ; p=0,779)$. Entretanto, as médias de uso de estratégias de regulação emocional foram mais elevadas no grupo de cuidadores solteiros e divorciados $(M=2,55 ; D P=0,24)$ do que no grupo de cuidadores casados ou em união estável $(M=1,90$; $D P=0,55)$ e a diferença entre médias foi estatisticamente significativa $(t[28]=2,426 ; p=0,038)$.

A quantidade de dias decorridos entre a internação do paciente e a comunicação do diagnóstico foi investigada em relação ao uso de estratégias de enfrentamento, a partir da divisão da amostra entre cuidadores que receberam o diagnóstico até uma semana após a internação, participantes comunicados sobre o diagnóstico entre oito e 15 dias após internação e acompanhantes que aguardaram mais de 16 dias pelo esclarecimento do diagnóstico. Cuidadores que aguardaram mais de 16 dias até comunicação do diagnóstico obti- 
veram médias mais elevadas em estratégias focalizadas na emoção $(M=2,78 ; D P=0,64)$ em relação a acompanhantes que receberam o diagnóstico na semana inicial de internação $(M=1,87 ; D P=0,56)$ e participantes que foram comunicados sobre o diagnóstico entre oito e 15 dias de internação $(M=2,31 ; D P=$ $0,5)$. As diferenças entre as médias dos grupos foram estatisticamente significativas $(F[2,27]=5,065 ; p=$ $0,014)$. Mais especificamente, a diferença entre médias de cuidadores que aguardaram mais de 16 dias pelo diagnóstico e acompanhantes que esperaram até uma semana foi estatisticamente significativa $(p=0,015)$, mas a diferença entre médias obtidas por cuidadores que aguardaram mais de 16 dias pelo diagnóstico e cuidadores que foram comunicados sobre o diagnóstico entre oito e 15 dias de internação não foi estatisticamente significativa $(p=0,467)$.

$\mathrm{Na}$ primeira e terceira fases do estudo, não foram encontradas correlações estatisticamente significativas entre a idade do participante e as categorias de estratégias de enfrentamento. Entretanto, na segunda fase do estudo, análises de correlação bivariada de Pearson indicaram que a idade do cuidador esteve correlacionada moderadamente, de forma positiva e estatisticamente significativa, à busca por suporte social, conforme ilustrado na Figura 1.

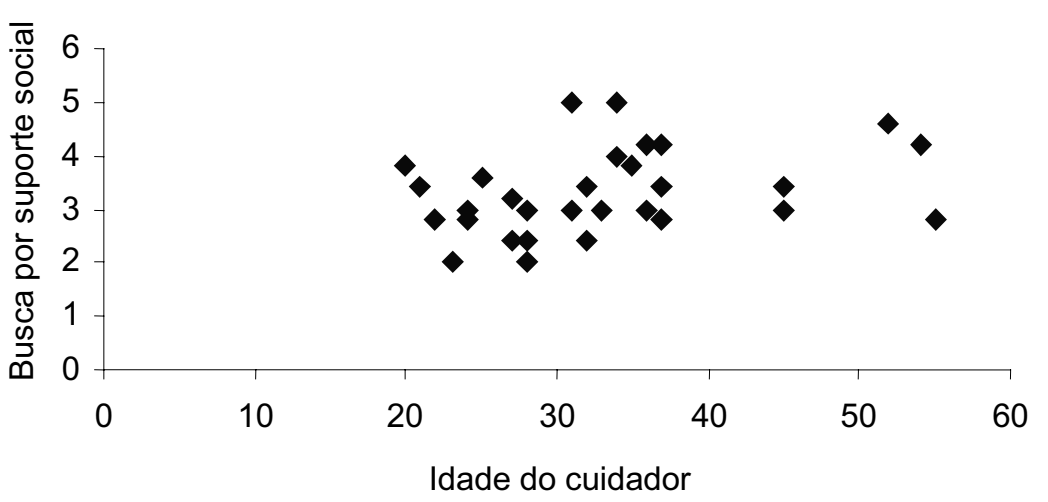

Figura 1. Correlação entre idade do cuidador e busca por suporte social.

Em relação ao uso de estratégias de enfrentamento, os participantes com escolaridade mais baixa (ensino fundamental incompleto e completo) obtiveram médias mais elevadas em estratégias focalizadas na emoção $(M=2,18 ; D P=0,68)$ quando comparados a participantes com escolaridade mais elevada - ensino médio completo ou incompleto e ensino superior - $(M=$ $1,58 ; D P=0,43)$ com diferença entre médias estatisticamente significativa $(t[28]=2,954 ; p=0,007)$.

$\mathrm{Na}$ primeira e segunda etapas da pesquisa, não foram encontradas associações estatisticamente significativas entre sexo dos participantes e estratégias de enfrentamento adotadas. Entretanto, na última fase do estudo, as médias de adoção de estratégias focalizadas no problema foram mais elevadas para homens $(M=$ $4,57 ; D P=0,30)$ em comparação a mulheres $(M=$ $4,16 ; D P=0,42)$ e as diferenças foram estatisticamente significativas $(t[28]=2,616 ; p=0,032)$. Em contrapartida, as médias relativas ao uso de estratégias de enfrentamento focalizadas na emoção foram mais elevadas para mulheres $(M=2,00 ; D P=0,67) \mathrm{em}$ comparação a homens $(M=1,49 ; D P=0,33)$ e a diferença também foi estatisticamente significativa $(t[28]$ $=2,533 ; p=0,026$ ).

Comparando grupos distintos quanto à renda mensal familiar, participantes de classes mais baixas (D e E) referiram escores mais elevados $(M=2,22 ; D P=$ $0,76)$ quanto ao uso de estratégias focalizadas na emoção, quando comparados a cuidadores de classe média $(M=1,64 ; D P=0,40)$ e a diferença entre médias foi estatisticamente significativa $(t[28]=2,516 ; p$ $=0,021)$. Entretanto, as pontuações nos outros fatores da EMEP foram similares entre os dois grupos e sem diferenças estatisticamente significativas para estratégias focalizadas no problema $(t[28]=0,128 ; p=$ $0,899)$, práticas religiosas e pensamentos fantasiosos $(t[28]=0,515 ; p=0,611)$ e busca por suporte social $(t[28]=0,316 ; p=0,755)$. 


\section{Estratégias de enfrentamento adotadas em cada fase de pesquisa}

Em relação às estratégias de enfrentamento relatadas em cada fase, a Figura 2 mostra a concentração de respostas nos fatores da EMEP, em cada fase do estudo.

$\mathrm{Na}$ primeira etapa do estudo, o Fator 3 (enfrentamento focalizado em práticas religiosas e pensamento fantasioso) foi priorizado pelos participantes $(M=$ $4,12 ; D P=0,51)$, seguido do Fator 1, relativo ao enfrentamento focalizado no problema $(M=3,89 ; D P$ $=0,64)$ e do Fator 4 (busca de suporte social, $M=$ 3,$68 ; D P=0,81$ ). O Fator 2 (enfrentamento focalizado na emoção) obteve menor pontuação, com média mais baixa do que os outros fatores $(M=2,06 ; D P=0,62)$. Essa distribuição entre as preferências por cada um dos fatores se repetiu nas etapas seguintes do estudo. $\mathrm{Na}$ segunda fase, o Fator 3 também foi priorizado $(M=3,93 ; D P=0,62)$, seguido pelo Fator $1(M=$ $3,89 ; D P=0,56)$, Fator $4(M=3,32 ; D P=0,80) \mathrm{e}$, por último, Fator $2(M=1,90 ; D P=0,65)$. Na última fase do estudo, o Fator 3 obteve médias mais altas $(M=$ $4,25 ; D P=0,62)$, seguido pelo Fator $1(M=4,23 ; D P$ $=0,43)$, Fator $4(M=3,49 ; D P=0,80)$ e Fator $2(M=$ $1,92 ; D P=0,66)$. A Figura 3 ilustra a distribuição dos fatores categorizados pela EMEP, ao longo das três fases do estudo.

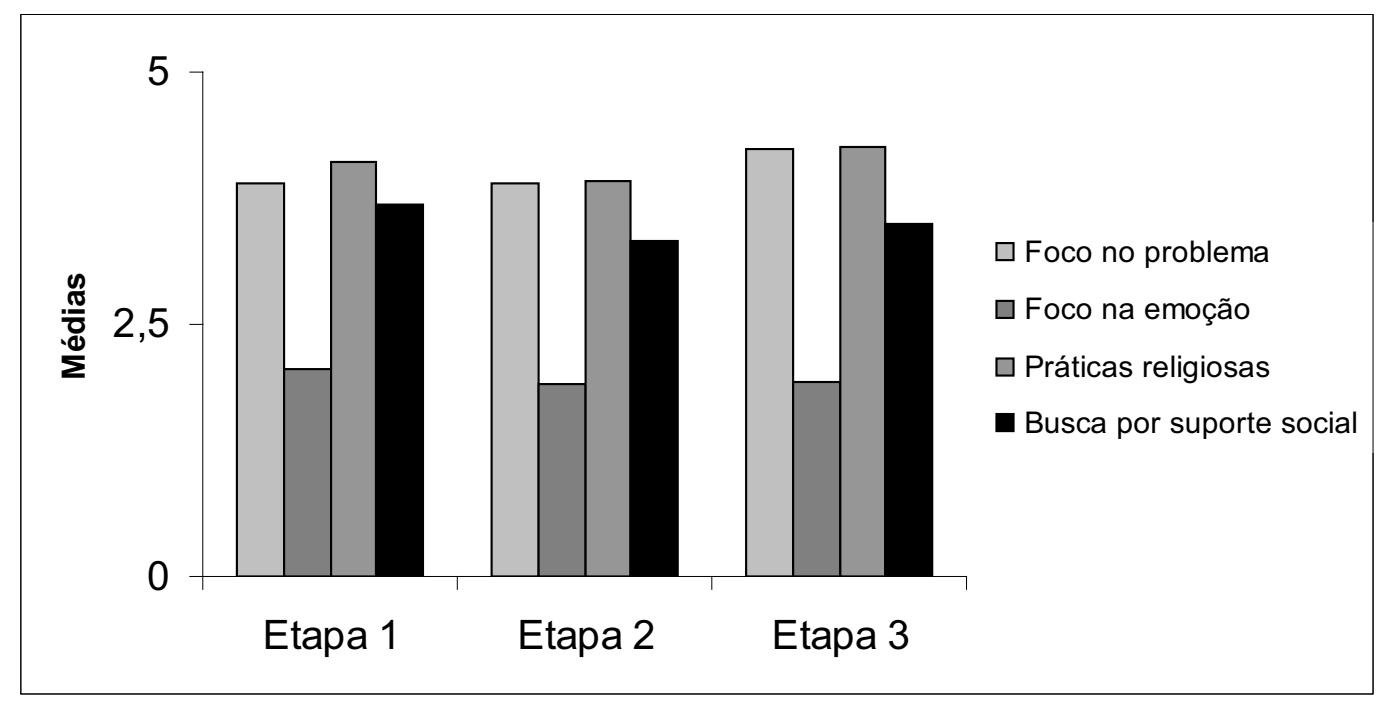

Figura 2. Médias nos fatores da EMEP em cada fase do estudo.

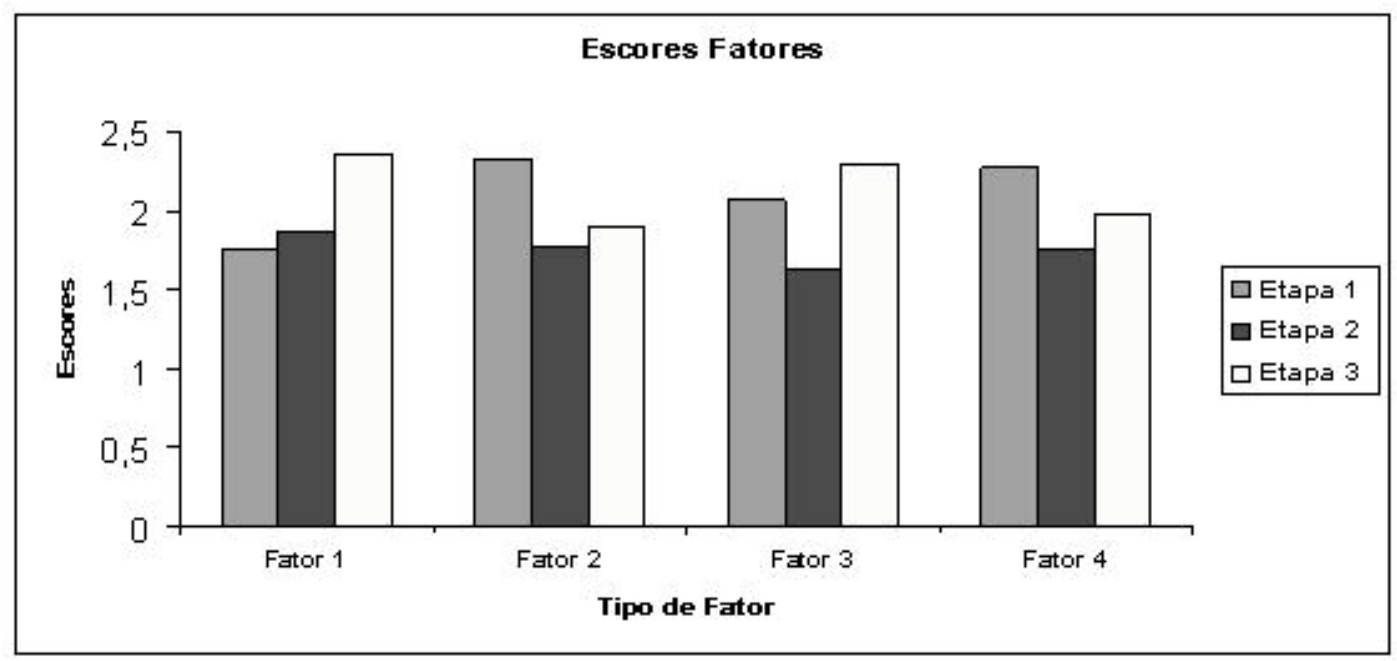

Figura 3. Escores nos fatores da EMEP ao longo das três fases do estudo. 
Em relação ao Fator 1 (enfrentamento focalizado no problema), os escores aumentaram ao longo das três fases e a diferença foi estatisticamente significativa $(p=0,008462)$. Pontuações relativas ao Fator 2 (enfrentamento focalizado na emoção) diminuíram significativamente ao longo das três fases $(p=$ 0,04391). Entre a segunda e terceira fases, houve um pequeno aumento nos escores, interpretado estatisticamente como uma continuação do processo de diminuição nas médias, confirmando a curva de decréscimo das pontuações relativas ao segundo fator, ao longo do tratamento.

Os escores do Fator 3 (enfrentamento focalizado em busca de práticas religiosas e pensamento fantasioso) e do Fator 4 (busca de suporte social) caracterizaram-se por um padrão semelhante, estatisticamente não significativo: diminuição entre a primeira e segunda fases e aumento entre a segunda e terceira etapas do estudo. Desta forma, as pontuações do Fator 3 não aumentaram nem decresceram ao longo das três etapas $(p=0,1831)$, bem como os escores do Fator 4 $(p=0,1237)$.

\section{Resultados apontados a partir de análises das entrevistas}

As respostas obtidas na primeira entrevista (referente à segunda etapa da pesquisa), sobre a adoção de estratégias de enfrentamento, foram classificadas segundo os Fatores indicados na EMEP. A respeito de estratégias de enfrentamento focalizadas no problema, três participantes descreveram comparar a condição do filho a situações piores, um cuidador destacou comparação a situações melhores e dois ressaltaram, como estratégia ativa, que não faziam comparação com outros casos ("cada caso é um caso, não comparo").

Em relação às ações práticas diretas ao tratamento, o planejamento foi referido por quatro cuidadores (" $a$ gente arruma tudo um dia antes, pra não esquecer papel, documento, receita, planejo tudo antes"). A busca por informações foi destacada por 13 cuidadores ("uma coisa que eu não sei, escrevo, na hora que chego no médico, eu pergunto"). Entre as fontes de obtenção de informação, os participantes relataram, principalmente, a equipe médica, além de outros profissionais do hospital, mães de outras crianças, internet e livros.

Os cuidadores também mencionaram ações dirigidas ao paciente e a outros filhos como estratégias de enfrentamento. Houve cinco relatos de monitoramento do paciente ("o que os médicos falam a gente faz, olhando, observando"), oito participantes descreveram conversar com a criança e explicar a situação ("tento explicar da melhor maneira") e houve um relato sobre conversar e explicar a situação para os irmãos do paciente. Outras estratégias de enfrentamento focalizadas no problema incluíram a necessidade de encarar a situação, com 15 relatos ("já encarei a doença de frente") e conformar-se, com três respostas ("o que me levou a aceitar foi a realidade de que não existe outro jeito").

Relatos de enfrentamento classificados como focalização na emoção revelaram que 16 participantes utilizaram o autocontrole ("tento achar o autodomínio"). Chorar e/ou protestar foi registrado em sete respostas e um cuidador destacou uso de práticas de relaxamento e ações meditativas. Três participantes relataram não expressar seus sentimentos à criança ou adolescente ("tento me controlar, sufocando tudo dentro de mim") e uso de negação foi referido por dois cuidadores ("você não acredita que é com você"). A maior parte dos participantes (27 cuidadores) enfatizou a importância de práticas de espiritualidade ("procurei a Deus") e o uso de crenças e pensamentos positivos foi referido por 12 participantes ("estou otimista que vai dar tudo certo").

A busca por suporte social emocional foi mencionada por 14 cuidadores ("minha vizinha, o filho dela teve esse problema, ela conversa comigo"). Treze participantes relataram busca por suporte instrumental ("transporte, no começo tava dificil, mas a gente tem amigo e eles ajudam"). As principais fontes de suporte envolveram familiares, amigos, vizinhos, mães de outras crianças e adolescentes em tratamento e a equipe de saúde.

Diversos exemplos de exigências do tratamento foram mencionados. Maior atenção e afetuosidade à criança foram destacadas por 11 participantes ("muita atenção, compreensão"). Nove cuidadores relataram necessidade de maior autocontrole e 11 participantes referiram o monitoramento da criança ("o cuidado triplicou, remédio, comida"). Oito cuidadores enfatizaram adaptações financeiras e mais gastos, somados a alterações no emprego, como desafiadores ("vir de carro, antes não tinha esse gasto").

$\mathrm{Na}$ segunda entrevista, correspondente à última etapa da pesquisa, os relatos também foram categorizados segundo a classificação proposta pela EMEP. Em relação a estratégias de enfrentamento focalizadas no problema, houve um relato de comparação a casos piores, outra descrição de confrontar com exemplos melhores e duas referências a comportamentos de não 
comparar com outros casos. Ações práticas diretas ao tratamento envolveram sete relatos de planejamento, uma referência à aprendizagem sobre procedimentos e uma descrição do uso de práticas complementares ("às vezes dou algum remédio para ele, caseiro"). Buscar informações foi mencionado por 14 cuidadores ("qualquer dúvida eu tiro com o médico, pergunto") e as principais fontes para obtenção de informações foram os membros da equipe médica, jornais, Internet e mães de outras crianças em tratamento.

O monitoramento do paciente foi destacado por sete participantes ("medicação eu nunca falhei") e explicar ou conversar com a criança, ou adolescente, foi descrito como estratégia de enfrentamento por quatro cuidadores; além disso, três participantes ressaltaram a prática de conversar e explicar aos irmãos saudáveis. Outras categorias de estratégias de enfrentamento focalizadas no problema incluíram 13 relatos sobre a necessidade de "encarar" ("tem que enfrentar, cada dificuldade eu enfrento de cara") e cinco participantes referiram conformar-se ("tive que acostumar à força").

Em relação a estratégias de enfrentamento focalizadas na emoção, 12 participantes mencionaram uso de autocontrole e houve dois relatos de choro e/ou protesto. Práticas de relaxamento e/ou meditação foram referidas por um cuidador e o esforço para não expressar sentimentos foi relatado por cinco participantes. Dois cuidadores descreveram uso de negação ("até hoje não acredito que ela tem essa doença") e três participantes descreveram uso de práticas de distração. Houve dois relatos de aumento da ingestão alimentar para controle de manifestações de ansiedade. $O$ foco na espiritualidade foi destacado por 24 participantes ("rezo, apego com a religião") e dez cuidadores ressaltaram a importância de crenças e pensamentos positivos ("sempre otimista"). A busca por suporte emocional foi mencionada em 20 relatos e a busca por suporte instrumental foi referida por 14 participantes ("quem ajudou foi vizinhos, amigos, com transporte, alimentação").

Em relação a exigências do tratamento, foram relatados quatro exemplos de necessidade de maior atenção e/ou afetuosidade para a criança ou adolescente ("exigiu afeição, carinho"). Dez participantes mencionaram maior paciência e autocontrole ("tenho que me controlar") e 11 cuidadores destacaram maior monitoramento do paciente ("observando o tempo todo, um cuidado maior"). Adaptações financeiras e necessidade de maiores gastos foram referidos em seis relatos ("a maior dificuldade é dinheiro") e três cuidadores destacaram a necessidade de conhecer a cidade para se locomover.

\section{DISCUSSÃO}

A distribuição de escores dos Fatores indicados pela EMEP corrobora os resultados do estudo de R. A. Fernandes, A. R. R. Santos e M. C. O. S. Miyazaki (comunicação pessoal sobre estudo realizado em 2006, 13 de fevereiro de 2008), que indicaram a preferência dos participantes por estratégias de enfrentamento focalizadas em práticas religiosas, seguidas pela focalização no problema, busca por suporte social e, finalmente, estratégias focalizadas na emoção. Além disso, em todas as fases do estudo, o Fator 3 (uso de práticas religiosas e pensamento fantasioso) correspondeu às médias mais elevadas, em comparação aos outros Fatores, refletindo alguns dados indicados na literatura sobre a focalização em estratégias religiosas (Elkin \& cols., 2007; Enskär \& cols., 1997; Goldbeck, 1998; Greening \& Stoppelbein, 2007; Munet-Vilaró, 1984; Norberg \& cols., 2005; Patistea, 2005; Patistea \& cols., 2000). Esse resultado pode ser influenciado pela cultura brasileira, caracterizada pela presença marcante da espiritualidade e crenças transcendentais. Além disso, a relativa incontrolabilidade da resposta clínica ao tratamento também pode influenciar a preferência por estratégias de cunho espiritualista, na medida em que os cuidadores podem atribuir o locus de controle da situação (e a resposta clínica) a fatores externos relacionados à ação transcendental, fora de controle direto.

As mudanças na adoção de estratégias de enfrentamento indicam o acompanhamento do processo de enfrentamento como condição antecedente ao planejamento sistemático de intervenções psicológicas que atendam às necessidades psicossociais de pais/familiares e pacientes. Tais alterações em estratégias de enfrentamento enfatizam, ainda, a importância do acompanhamento sobre as estratégias de enfrentamento priorizadas, para planejamento de intervenções psicossociais que possam diminuir custos e demandas do tratamento.

Ao longo do estudo, o aumento da ocorrência de estratégias referidas no Fator 1 (comportamentos focalizados no problema) pode fundamentar-se no cumprimento de instruções recebidas da equipe de saúde quanto a cuidados médicos relativos à higiene, limpeza, ausência de contato com indivíduos doentes, dieta adequada e medicação, que se constituem ações focalizadas no domínio do estressor ou resolução do problema. Estratégias de enfrentamento focalizadas na resolução do problema, relatadas em entrevista, referiram comportamentos não abordados pela EMEP, mas 
indicados na literatura, tais como aprender sobre a doença e procedimentos médicos, explicar e conversar com o paciente e/ou irmãos saudáveis, "encarar" a situação (comportamento caracterizado, na realidade, pela ausência de comportamentos de fuga e esquiva), conformar-se, monitorar o paciente, comparar a casos melhores ou não utilizar a comparação e adotar práticas terapêuticas complementares (Holm \& cols., 2003; Patistea, 2005; Patistea \& cols., 2000).

Em relação ao uso de estratégias focalizadas na emoção, os relatos indicaram uma categoria não abordada na EMEP (mencionada no estudo de Norberg \& cols., 2005), referente à utilização de técnicas de relaxamento e/ou meditação. A categoria relativa à espiritualidade e expressão de crenças e pensamentos positivos indicou resultados importantes: em ambas as entrevistas, praticamente todos os participantes referiram adotar práticas espiritualistas e cerca de metade dos cuidadores referiu uso de crenças ou pensamentos positivos, corroborando dados obtidos na aplicação da EMEP, que apontam essa categoria de enfrentamento como a mais adotada nas três etapas do estudo. Tais resultados apontam para a importância da utilização de metodologias e análises combinadas, quantitativas e qualitativas, para uma apreensão fidedigna da situação vivenciada pelo participante.

Destaca-se que a EMEP apresenta vantagens em sua utilização: a aplicação no início do tratamento pode identificar precocemente casos que necessitem de atendimento individual, atenção especial da equipe ou intervenção em crise, viabilizando o tempo disponível, além de localizar crenças disfuncionais, estratégias de enfrentamento não adaptativas e mesmo dúvidas que podem não surgir durante consultas ou demorar a ser relatadas em atendimento.

É importante salientar uma consequência relevante advinda da aplicação da EMEP, relativa ao fornecimento de dicas sobre comportamentos do cuidador, incentivo à auto-observação e fornecimento de contextos propícios para reestruturação cognitiva a partir da apresentação de possibilidades de comportamento diferentes do comportamento percebido. Em outras palavras, o uso da EMEP se constitui, por si só, uma forma de intervenção, possibilitando aos cuidadores uma reflexão e avaliação acerca de seus próprios comportamentos, muitas vezes não sinalizados em outros contextos. As dicas oferecidas podem auxiliar o participante a conhecer melhor seu comportamento, proporcionando a modificação de padrões comportamentais e exploração de outras estratégias.

\section{CONSIDERAÇÕES FINAIS}

Os resultados obtidos apontam algumas informações relevantes à prática clínica dos profissionais de saúde que atuam na área da psico-oncologia pediátrica. O esforço para compreender a vivência de cada cuidador ao longo do tratamento é fundamental para o planejamento de intervenções que possam, efetivamente, alterar as contingências estressantes do tratamento, de modo a minimizar custos comportamentais e cognitivos tanto para o paciente quanto para seus familiares.

Outras pesquisas tornam-se necessárias para esclarecer aspectos funcionais importantes sobre o enfrentamento de condições adversas em contexto de tratamento pediátrico, especialmente durante diferentes fases da doença. Torna-se essencial o acompanhamento de todo o processo de diagnóstico, tratamento e encerramento do protocolo, em delineamento longitudinal. Destaca-se a necessidade de mais estudos com análises complementares, em âmbito nacional e multicêntrico, que focalizem as estratégias de enfrentamento, o ajustamento psicológico e a adaptação de cuidadores de crianças e adolescentes frente ao diagnóstico e tratamento.

Pesquisas futuras sobre estratégias de enfrentamento em contexto onco-hematológico pediátrico devem incluir o paciente e também seus familiares, em uma abordagem global e sistêmica. Pesquisas focalizadas nas equipes de atendimento também são essenciais, a fim de compreender como os médicos, psicólogos, nutricionistas, enfermeiros e demais profissionais lidam com desafios e demandas em seus atendimentos. Também são necessárias pesquisas para construção de outros instrumentos de medida mais adequados ao contexto onco-hematológico pediátrico, voltados para crianças, adolescentes e adultos, que incluam itens referentes a procedimentos médicos invasivos, efeitos colaterais, expectativas de recaída e cura e outros elementos próprios do contexto.

De modo especial, a mudança nas estratégias de enfrentamento adotadas ao longo dos meses iniciais de tratamento enfatiza que o acompanhante de uma criança, ou adolescente, em tratamento é, também, um indivíduo em processo de desenvolvimento humano. Sendo assim, deve ser considerado um elemento fundamental e ativo na dinâmica paciente-familiar-profissional de saúde, não apenas um informante sobre condições clínicas do paciente e responsável por cuidados intrínsecos ao tratamento. A atuação preventiva com os cuidadores pediátricos tem influência 
direta sobre a sua vivência e percepção do tratamento e ainda condiciona as relações que se estabelecem entre os familiares, o paciente e o ambiente de cuidados médico-hospitalar, contribuindo para a melhoria da qualidade de vida de pacientes, familiares e, inclusive, dos profissionais de saúde.

\section{REFERÊNCIAS}

Beltrão, M. R. L. R., Vasconcelos, M. G. L., Pontes, C. M., \& Albuquerque, M. C. (2007). Câncer infantil: Percepções maternas e estratégias de enfrentamento frente ao diagnóstico. Jornal de Pediatria, 83(6), 562-566.

Castro, E. K., \& Piccinini, C. A. (2002). Implicações da doença orgânica crônica na infância para as relações familiares: Algumas questões teóricas. Psicologia: Reflexão e Crítica, 15(3), 625-635.

Clarke, J. (2004). Whose power/authority/knowledge? Conundrums in the experiences of parents whose children have cancer. Social Work in Health Care, 40(2), 13-35.

Clarke, J. N., \& Fletcher, P. (2003). Communication issues faced by parents who have a child diagnosed with cancer. Journal of Pediatric Oncology Nursing, 20(4), 175-191.

Clarke-Steffen, L. (1997). Reconstructing reality: Family strategies for managing childhood cancer. Journal of Pediatric Nursing, 12(5), 278-287.

Costa Junior, A. L. (2005). Psicologia da saúde e desenvolvimento humano: $\mathrm{O}$ estudo do enfrentamento em crianças com câncer e expostas a procedimentos médicos invasivos. Em M. A. Dessen \& A. L. Costa Junior (Orgs.), A ciência do desenvolvimento humano: Tendências atuais e perspectivas futuras (pp. 171189). Porto Alegre: Artmed.

Eiser, C., Havertnans, T., \& Elser, J. R. (1995). Parents' attributions about childhood cancer: Implications for relationships with medical staff. Child: Care, Health and Development, 21(1), 31-42.

Elkin, T. D., Jensen, S. A., McNeil, L., Gilbert, M. E., Pullen, J., \& McComb, L. (2007). Religiosity and coping in mothers of children diagnosed with cancer: An exploratory analysis. Journal of Pediatric Oncology Nursing, 24(5), 274-278.

Enskär, K., Carlsson, M., Golsäter, M., Hamrin, E., \& Kreuger, A. (1997). Parental reports of changes and challenges that result from parenting a child with cancer. Journal of Pediatric Oncology Nursing, 14(3), 156-163.

Felder-Puig, R., Gallo, A., Waldenmair, M., Gadner, H., \& Topf, R. (2004). The TCCS: A short measure to evaluate treatmentrelated coping and compliance in hospitalized childhood cancer patients and their primary caregivers. Support Care Cancer, 12(1), 41-47.

Folkman, S., \& Lazarus, R. S. (1985). If it changes it must be a process: Study of emotion and coping during three stages of a college examination. Journal of Personality and Social Psychology, 48(1), 150-170.

Goldbeck, L. (1998). Familial coping with cancer in childhood and adolescence. Possibilities for standardized assessment with a self-assessment method: Results of an empirical pilot study.
Praxis der Kinderpsychologie und Kinderpsychiarier,47(8), 552-573.

Greening, L., \& Stoppelbein, L. (2007). Brief report: Pediatric cancer, parental coping style, and risk for depressive, posttraumatic stress, and anxiety symptoms. Journal of Pediatric Psychology, 32(10), 1272-1277.

Herman, A. R. S. (2007). Câncer pediátrico: Impacto de intervenção psicoeducacional sobre enfrentamento e práticas parentais. Tese de doutorado não-publicada, Faculdade de Medicina de São José do Rio Preto.

Hoekstra-Weebers, J. E. H. M., Jaspers, J. P. C., Kamps, W. A., \& Klip, E. C. (1998). Marital dissatisfaction, psychological distress, and the coping of parents of pediatric cancer patients. Journal of Marriage and the Family, 60(4), 1012-1021.

Hoekstra-Weebers, J. E. H. M. Jaspers, J. P. C., Kamps, W. A., \& Klip, E. C. (2001). Psychological adaptation and social support of parents of pediatric cancer patients: A prospective longitudinal study. Journal of Pediatric Psychology, 26(4), 225-235.

Holm, K. E., Patterson, J. M., \& Gurney, J. G. (2003). Parental involvement and family. Centered care in the diagnostic and treatment phases of childhood cancer: Results from a qualitative study. Journal of Pediatric Oncology Nursing, 20(6), 301313.

James, K., Keegan-Wells, D., Hinds, P. S., Kelly, K. P., Bond, D., Hall, B., Mahan, R., Moore, I. M., Roll, L., \& Speckhart, B. (2002). The care of my child with cancer: Parent's perceptions of caregiving demands. Journal of Pediatric Oncology Nursing, 19(6), 218-228.

Kars, M. C., Duijnstee, M. S. H., Pool, A., Delden, J. J. M., \& Grypdonck, M. H. F. (2008). Being there: Parenting the child with acute lymphoblastic leukaemia. Journal of Clinical Nursing, 17(12), 1553-1562.

Keegan-Wells, D., James, K., Stewart, J. L., Moore, I. M., Kelly, K. P., Moore, B., Bond, D., Diamond, J., Hall, B., Mahan, R., Roll, L., \& Speckhart, B. (2002). The care of my child with cancer: A new instrument to measure caregiving demand in parents of children with cancer. Journal of Pediatric Nursing, 17(3), 201-210.

Klassen, A., Raina, P., Reineking, S., Dix, D., Pritchard, S., \& O'Donnell (2007). Developing a literature base to understand the caregiving experience of parents of children with cancer: A systematic review of factors related to parental health and wellbeing. Support Care Cancer, 15(7), 807-818.

Kohlsdorf, M. (2008). Análise das estratégias de enfrentamento adotadas por pais de crianças e adolescentes em tratamento de leucemias. Dissertação de mestrado não-publicada, Universidade de Brasília.

Lazarus, R. S., \& Folkman, S. (1984). Stress, appraisal, and coping. New York: Springer.

McGrath, P. (2002). Beginning treatment for childhood acute lymphoblastic leukemia: Insights from the parents' perspective. Oncology Nursing Forum, 29(6), 988-996.

McGrath, P., Paton, M. A., \& Huff, N. (2005). Beginning treatment for pediatric acute myeloid leukemia: The family connection. Issues in Comprehensive Pediatric Nursing, 28(2), 97114. 
Motta, A. B., \& Enumo, S. R. F. (2002). Brincar no hospital: Câncer infantil e avaliação do enfrentamento da hospitalização. Psicologia, Saúde e Doenças, 3(1), 23-41.

Munet-Vilaró, F. (1984). Coping strategies and adaptation to childhood cancer of Puerto Rican families. Washington: Ann Arbor.

Nascimento, L. C. Rocha, S. M. M., Hayes, V. H., \& Lima, R. A. G. (2005). Crianças com câncer e suas famílias. Revista da Escola de Enfermagem da USP, 39(4), 469-474.

Norberg, A. L., Lindblad, F., \& Boman, K. K. (2005). Coping strategies in parents of children with cancer. Social Science \& Medicine, 60(5), 965-975.

Patistea, E. (2005). Description and adequacy of parental coping behaviors in childhood leukaemia. International Journal of Nursing Studies, 42(3), 283-296.

Patistea, E., Makrodimitri, P., \& Pantelli, V. (2000). Greek parents' reactions, difficulties and resources in childhood leukae$\mathrm{mia}$ at the time of diagnosis. European Journal of Cancer Care, 9(2), 86-96.

Rodrigues, M. A., Rosa, J., Moura, M. J., \& Baptista, A. (2000). Ajustamento emocional, estratégias de coping e percepção da doença em pais de crianças com doença do foro oncológico. Psicologia, Saúde e Doenças, 1(1), 61-68.

Seidl, E. M. F., Tróccoli, B. T., \& Zannon, C. M. L. C. (2001). Análise fatorial de uma medida de estratégias de enfrentamento. Psicologia: Teoria e Pesquisa, 17(3), 225-234.

Shiota, C. M., Santos, A. R. R., \& Miyazaki, M. C. O. S. (2004). Problemas de comportamento em crianças com câncer: O papel dos pais. Em M. Z. S. Brandão, F. C. S. Conte, F. S. Brandão,
Y. K. Ingberman, C. B. Moura, V. M. Silva \& S. M. Oliane (Orgs.), Sobre comportamento e cognição: Vol., 14. Estendendo a psicologia comportamental e cognitiva aos contextos da saúde, das organizações, das relações pais e filhos e das escolas (pp. 261-266). São Paulo: ESETec.

Silva, S., Pires, A., Gonçalves, M., \& Moura, M. J. (2002). Cancro infantil e comportamento parental. Psicologia, Saúde \& Doenças, 3(1), 43-60.

Sloper, P. (2000). Predictors of distress in parents of children with cancer: A prospective study. Journal of Pediatric Psychology, 25(2), 79-91.

Steele, R. G., Long, A. L., Reddy, K. A., Luhr, M., \& Phipps, S. (2003). Changes in maternal distress and child-rearing strategies across treatment for pediatric cancer. Journal of Pediatric Psychology, 28(7), 447-452.

Tarr, J., \& Pickler, R. H. (1999). Becoming a cancer patient: A study of families of children with acute lymphocytic leukemia. Journal of Pediatric Oncology Nursing, 16(1), 44-50.

Trask, P. C., Paterson, A. G., Trask, C. L., Bares, C. B., Birt, J., \& Maan, C. (2003). Parent and adolescent adjustment to pediatric cancer: Associations with coping, social support, and family function. Journal of Pediatric Oncology Nursing, 20(1), 36-47.

Young, B., Dixon-Woods, M., Findlay, M., \& Heney, D. (2002). Parenting in a crisis: Conceptualizing mothers of children with cancer. Social Science \& Medicine, 55(10), 1835-1847.

Recebido: 17/12/2008

Última revisão: $31 / 05 / 2009$ Aceite final: 24/07/2009

\section{Sobre os autores:}

Marina Kohlsdorf: Mestrado em Processos de Desenvolvimento Humano de Saúde pela Universidade de Brasília.

Áderson Luiz da Costa Junior: Pós-Doutorado pela Universidade Estadual de Campinas. Professor do Instituto de Psicologia da Universidade de Brasília.

Endereço para correspondência: marinak@unb.br. 